\title{
DE LOS DIEZ A LOS VEINTE AÑOS (1678-1688)
}

\section{Giorgio Giacometti Pinton \\ (Connecticut, USA)}

\begin{abstract}
RESUMEN: En este trabajo el Autor repasa los momentos principales claves de su comprensión de Vico. Se centra en recrear con un bello relato los diez años de la infancia a la juventud del napolitano para, a través de ese recorrido hasta los veinte años de edad, ir reconstruyendo a la vez que una interpretación de las raíces de la formación de Giambattista, también momentos de la propia experiencia vital del Autor.

Palabras ClAVE: Vico, $350^{\circ}$ Aniversario, niñez, adolescencia, biografía, filosofía, formación, G.G. Pinton.
\end{abstract}

From ten to twenty years (1678-1688)

ABSTRACT: In this work the Author reviews the key moments of his understanding of Vico. It aims at recreating with a beautiful story the ten years that pass between the Neapolitan's childhood and youth. Through this journey up to his twenty years of age, he reconstructs both an interpretation of the roots of Giambattista's formation, and some moments of the Author's own life experience.

KEYWORDS: Vico, 350th Anniversary, childhood, adolescence, biography, philosophy, formation, G.G. Pinton.

\section{Dai dieci ai vent'anni (1678-1688)}

RIASSUNTO: In questo lavoro l'Autore ripercorre i momenti chiave della sua comprensione del pensiero di Vico. Si sofferma sul ricreare attraverso un bel racconto i dieci anni che vanno dall'infanzia alla gioventù napoletana per ricostruire, attraverso questo percorso che arriva fino ai vent'anni di età del pensatore, un'interpretazione delle radici della formazione di Giambattista e, al tempo stesso, i momenti dell'esperienza di vita dell'Autore.

Parole ChiAve: Vico, $350^{\circ}$ Anniversario, fanciullezza, adolescenza, biografia, filosofia, formazione, G.G. Pinton.

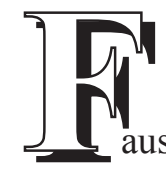

austo Nicolini, en La Giovinezza di Giambattista Vico (1932), relató el cambio que se produjo en la personalidad del «fanciulletto» tras caer de la escalera de la librería de su padre en 1675, por cuya caída, de acuerdo con un tal Doctor Cesare Basso, se pronosticó que Giambattista debería haber sufrido «o morte o perenne idiozia». Nada de eso ocurrió. Sin embargo, y afortunadamente, Nicolini narró:

Este artículo responde a una invitación expresa por parte de la Dirección de la Revista para este volumen especial por el $350^{\circ}$ Aniversario del nacimiento de G. Vico, habiendo superado los criterios de valoración y del proceso de aceptación. 
«Tuttavia non fu più quello di una volta. Divenuto e restato sempre nervosissimo, già da allora cominciò a mostrare qualcuna di quelle che furono poi note predominanti del suo carattere: un'affettività squisita; una sensibilità quasi morbosa; una semplicità, un'ingenuità, un candore angelici; una generosità eroica; una tendenza a una malinconia talora dolce e loquace, tal'altra cupa e taciturna; un bisogno assillante di vivere con se medesimo, esaminandosi, studiandosi e sovente torturandosi; una coscienza della dignità umana e della serietà della vita che, nel grado eminente in cui egli la possedè, fu nella Napoli del suo tempo più unica che rara; una volontà inflessibile e una perduranza ferrea, che consentirono a quella sua carne interna vere orgie di lavoro, da cui un temperamento più sano ma meno volitivo sarebbe forse uscito distrutto; un voler sempre al concepimento e al proposito far seguire immediata l'azione; una nervosa insofferenza di qualsiasi ostacolo eil non saper resistere qualche volta alla tentazione di saltarli a piè pari anzichè demolirli con lunga e paziente fatica; pur con abitudini di grande ordine nella vita pratica, una certa indisciplina mentale, che lo induceva troppo spesso a violare nel campo degli studi la legge economica del minimo mezzo; un'acre causticità, manifestantesi talvolta in motti meramente scherzosi, più spesso in sarcasmi amari; un'opinione altissima del proprio valore, stranamente commista con una verginale e talora caparbia modestia, contrastante a sua volta con un inappagato e non dissimulato desiderio di lode; e, con una permalosità troppo ombrosa, ma anche con la più compiuta assenza d'invidia e di livore, un'impetuosità e una collericità a volte spaventose». ${ }^{1}$

Naturalmente, dicho de un modo general, este retrato representa al Vico adulto, aunque tanto las cualidades positivas como las negativas ya estaban presentes en el joven, y algunas se desarrollaron desde el momento en que, tras tres años de convalecencia, regresó a la escuela.

Estas palabras de Nicolini describieron al Vico joven, al adulto y al anciano, con las cuales he vivido durante medio siglo en mi mente. Conocerle bien es la clave para comprender sus logros y trabajos, ya sean de ciencia, filosofía, poesía, o de historia.

En noviembre de 1678, Giambattista Vico se reencontró con sus compañeros a su regreso en la Escuela de Gramática. En casa, realizó con tal rapidez las tareas que le remitió su profesor que su padre pensó que estaba siendo descuidado, de modo que fue a la escuela para preguntarle al profesor si su hijo estaba haciendo las tareas 
como las haría un buen estudiante. Siendo afirmativa la respuesta, le pidió que doblase los deberes de su hijo. El profesor objetó a esto indicando que el ritmo de trabajo de Giambattista debía estar acompasado al del resto de estudiantes. Pero entonces el joven alumno solicitó sin pudor que se le permitiese pasar al siguiente curso, prometiendo que él se encargaría por sí mismo de adquirir la preparación que fuese precisa. Fue a partir de este momento cuando Giambattista comenzó a actuar como su propio profesor.

Sin embargo, en cuanto se le asignó un profesor que era todavía menos estimulante que el primero, el muchacho expresó su pesar a su padre, quien decidió inscribirle en la escuela de los jesuitas, la cual se encontraba a poco más de una cuadra de su casa. La escuela en cuestión estaba en Vicolo San Marcellino y había sido fundada 80 años antes por Roberta Carafa (1587-1603), esposa de Camillo Caracciolo (15631617), recordada por una inscripción en el patio del palacio de Cortile del Salvatore.

Esto siempre me ha recordado a mi formación independiente para el examen estatal de Latín que se requiere para obtener el certificado de admisión e inscripción en los pequeños cursos que se imparten en el Seminario del Duomo de Milán. Fue en el año 1938. Para mí fue un modo de salir de la pobreza fisica y mental, alejado de mi familia, y vivir en el Seminario del Duomo, donde pude estudiar y residir, y como compensación a ello se suponía que debía servir como monaguillo en las misas y otras ceremonias religiosas en la Gran Catedral Gótica en la época del Cardenal Alfredo Ildefonso Schuster (1880-1954), un estudioso especializado en historia monástica y temas litúrgicos.

En una fría mañana de octubre de 1680, Giambattista caminó hasta la escuela para comenzar el semestre en el programa de estudios básicos, que incluía tres cursos de gramática y dos de humanidades y retórica. Fue admitido en el segundo curso. Apenas durante sus primeros meses de asistencia, los padres jesuitas tuvieron ocasión de comprobar la altura de su talento. Hacia el final del semestre, los padres lo seleccionaron para competir con tres de los alumnos más dotados en un extraordinario ejercicio escolástico para obtener una plaza. Giambattista doblegó al primero de los tres, y el segundo se dio por vencido a mitad de la competición. Pero el tercero, favorecido por los padres, pasó al tercer curso con honores y quedó exonerado de realizar ese ejercicio. Giambattista, recibiendo esto como una afrenta personal, se enojó de tal manera ante este acuerdo que en abril de 1681 decidió dejar de frecuentar esta escuela jesuita para, de hecho, seguir aprendiendo por su cuenta.

Y así, se esforzó con tal presteza y entusiasmo que en octubre del mismo año ya había terminado el tercer curso de Gramática Latina gracias al De Institutione grammatica del jesuita portugués Manuel Álvarez (1526-1583). Encontró este texto y las Institutiones linguae graecae de Giacomo Gretser (1561-1623) en la tienda de su padre, el lugar donde pasaba casi todo su tiempo y en cuya trastienda pudo dedi- 
carse apaciblemente a sus estudios predilectos, humanidades y retórica. Entonces, prosiguió con fervor el programa de estudios superiores, que por lo general incluía dos o tres años de filosofía y cuatro de teología. Comenzó con lógica. Contó con la ayuda del padre jesuita Antonio del Balzo (1650-1725), un filósofo nominalista, quien le sugirió que estudiase a Petrus Hispanus (de Lisboa, m. 1277) y la Logica Magna oculta en las Summulae de Paulus Venetus (1372 ca.-1429), cuyo nombre real era Paolo Nicoletti de Udine. Así lo hizo: al atardecer se sentará en su escritorio rodeado de libros, donde pasará a menudo todo el tiempo hasta el amanecer.

Al abandonar a los profesores jesuitas, Vico habría tenido la fortuna de continuar rodeado por autores jesuitas de todos los temas. En 1952 estudié la Logica Maior como la Magna que Vico trató de seguir. Igual que él. Me gustaba estar rodeado de libros, sobre todo de libros con uno o dos siglos de antigüedad.

Su amada madre, tras levantarse, le pediría sin éxito que se fuese a la cama.

¡Resulta arduo abandonar un asunto en el que te hallas sumergido, sabiendo la dificultad que entraña recuperar una línea de pensamiento interrumpido!

En verano de 1682, Giambattista aún andaba enredado en la lógica de Crisipo, agotado y desalentado ante la falta de progresos. ¡Su mentor había errado a la hora de empujarle al estudio de una ciencia que estaba por encima de su edad! Su entusiasta mente juvenil era todavía lo suficientemente débil para ello. Abatido, molesto consigo mismo por las limitaciones impuestas por la naturaleza y desorientado acerca de qué hacer, Giambattista acabó por claudicar.

Durante un año y medio ayudó a su padre en la tienda, aprendiendo el cuidado y la custodia de los libros, y a veces a su madre y a sus hermanos pequeños en la casa. Cuando libraba de sus tareas, o en escapadas con otros jóvenes de la zona, practicaba su caligrafía copiando de libros. En ocasiones, ese grupo de jóvenes atravesaría la Via San Biagio dei Librai hasta Via Santa Chiara y cantaría bajo las ventanas de la casa que en su día fue de Giulia Gonzaga. Esta murió en 1556, aunque su belleza fue celebrada en los versos del Orlando Furioso de Ludovico Ariosto (1473-1533), versos que Vico había aprendido de memoria: «Giulia Gonzaga, che dovunque il piede / Volge, e dovunque i sereni occhj gira, / Non pure ogn' altra di beltà le cede, / Ma, come scesa dal ciel Dea, l'ammira. / [...]» (XLVI, 8].

En uno de sus estados melancólicos, cruzó la calle en soledad y con un libro bajo su brazo alcanzó la esquina de la Via dei Tribunali para hallar la paz en la Iglesia de San Lorenzo Maggiore, para allí leer páginas y páginas de la obra de Torquato Tasso (1544-1595), sentado en silencio junto a la quinta capilla de la derecha. Allí se encuentra la tumba de Giambattista Manso (1569-1645), Marqués de Villa, quien fundó en 1611 la Accademia degli Oziosi y sentía una veneración absoluta por el poeta Tasso, haciéndolo objeto de su continua prodigalidad. En el claus- 
tro del Monasterio de San Lorenzo conoció la Academia degli Infuriate, fundada en 1617 por Francesco Carafa d'Anzi, cuyo lema era Agitante calescimus illo, y la Academia de Oscuri, interesada en debates literarios y jurídicos, fundada por Paduano Guasco en 1679 con el lema Et latet et lucet, con una pintura del sol entre las nubes. Los Oziosi se reunían desde 1815 en la Iglesia de San Domenico Maggiore, a cuyo monasterio se accedía a través de una puerta sobre la cual se observaba la inscripción Schola Divi Thomae Aquinatis y donde fue inaugurada la Universidad de Nápoles por voluntad del rey Carlo I d'Angiò (1227-1285). Fue esta tierra, tan repleta de iniciativas e instituciones de enseñanza, sobre la que Vico caminó, recorriendo siempre con la mirada elevada su alrededor.

Stephen Hawking nunca dejó de recordarlo:

«recuerda levantar la vista hacia las estrellas y no bajarla a tus pies... Trata de dar sentido a cuanto veas y pregúntate qué es lo que hace que exista el universo. Sé curioso. Y por dificil que la vida pueda parecer, siempre habrá algo que puedas hacer y en lo que tener éxito. Lo único que hace falta es que no te rindas». ${ }^{2}$

A esa edad, siendo un adolescente liberado de la escolarización, Giambattista sin duda llegó a explorar Castel dell'Ovo con su grupo habitual de amigos, e iría a jugar a la playa rocosa, recogiendo conchas, observando a los pescadores remendando sus redes o limpiándolas. Él sabía cuándo se erigió Castel dell'Ovo como una fortaleza, fue él quien les dijo a los demás que antiguamente se decía que el poeta latino Lúculo tenía su villa en el mismo lugar donde se yergue la fortaleza. En otras ocasiones iban al norte siguiendo la curva que describe el golfo hasta Mergellina, la perla del golfo. Desde allí, mirando hacia el sur, podía contemplar el poder y la majestuosidad del Vesubio y del arco completo del golfo. Era una vista que les sobrecogía física y mentalmente a sus compañeros y a él, siendo tan sensible a los fenómenos naturales. Desde lo alto de la colina, en las callejuelas que se arropaban dentro de la alta muralla de los palacios entre los que vivían, solo podían mirar directamente hacia el cielo.

Su padre conocía a algunos jesuitas que a veces se detenían en la librería. Le dijo a su hijo que acudiese a Gesú Nuovo y preguntase por Giuseppe Ricci da Lecce (1650-1713). Así lo hizo, y Ricci, viendo el entusiasmo del joven y con la esperanza de suscitarle también interés por la orden de los jesuitas, aceptó con agrado introducir a Giambattista en la filosofía, primero de Platón y luego de Duns Scoto. A lo largo de tres meses continuó sus clases privadas con Ricci, a quien siempre reconoció como un hombre de profundas ideas, excepto por el hecho de que se estaba vol-

2. C.S. Monitor Weekly, 2 de abril 2018. 
viendo extremadamente meticuloso a la hora de empeñarse en explicaciones innecesarias. Él se mostraba más bien impaciente por nuevos conocimientos. En casa, combinando el tiempo del estudio con el dedicado a catalogar, ordenar y limpiar el polvo de los libros, también ayudaba a su madre y hermanos. ¡Cinco en total! Pero en uno de aquellos días de noviembre, cuando las calles de Nápoles se anegan, tomó la decisión de no dirigirse a la mansión jesuita para, en lugar de eso, y con el consentimiento de su padre, pasar más tiempo abajo en la tienda incluso antes de que abriese. En soledad y con impaciencia, miraba a su alrededor cuando atisbó una pila de libros en los que no había reparado hasta entonces. Había quince tomos y pensó que debía de tratarse de una nueva adquisición o que habrían sido llevados a la tienda por su valor. El autor era miembro de la "S.J." y los títulos le sorprendieron: Summa Theologica, Disputationes Metaphysicae, De virtute et statu Religionis, Defensio Fidei, De Divina Gratia. Cada obra tenía más de un tomo. Giambattista quedó tan cautivado e interesado que tomó justo el primero de los dos volúmenes de las Disputationes Metaphysicae y comenzó a leer. Horas más tarde, su padre regresó de un encargo a la tienda y encontró a su hijo inmerso en la lectura del libro. Tras comprobar de cuál se trataba, Antonio De Vico tuvo la oportunidad de explicarle a su hijo que el autor, Francisco Suárez (1548-1617), había sido el más importante teólogo y filósofo escolástico del siglo anterior. Giambattista, que nunca antes había recibido de su padre comentarios sobre libros, se vio abrumado por cuánto sabía a este respecto sobre el autor y el contenido del libro. Suárez enseñó en Salamanca, Valladolid, Granada y también en el Collegium Romanum de Roma. Suárez, dijo el padre, era claro en su escritura y tenía un estilo fácil de leer, pero al mismo tiempo hacía alarde de una elocuencia sin igual. Añadió que con esas características Suárez explicó toda la filosofía. ¡Esas fueron las razones por las que se había sentido tan atraído desde el mismo momento en que abrió el libro! Se vio abrumado de gozo y le pidió permiso a su padre para quedarse con los dos volúmenes hasta que los hubiese terminado. Así, de nuevo, no regresó a las clases en el "Gesú Nuovo" y pasó el tiempo en casa estudiando a Suárez. Su padre debió de olvidarse de aquellos dos volúmenes, porque Giambattista se los quedó durante todo un año.

¡Oh, cuán embelesado estoy ante la fascinación y la atracción y el misterio de los antiguos libros en latín!

Aquello ya fue a comienzos de 1684. Durante una de sus visitas a San Lorenzo Maggiore pasó cerca de la puerta que da paso al claustro y oyó voces que procedían de una de las habitaciones situadas en uno de los lados. Las celdas de los antiguos monjes. Pero en esta ocasión, cuando se aproximó, la celda estaba repleta de eminentes hombres de letras, ya que reconoció a algunos abogados, senadores y nobles de la ciudad, y sus voces resonaban dentro del claustro, de modo que pudo escuchar sin que se percataran de su presencia. Se sentó en el muro, bajo la línea de 
columnas que bordean el jardín central y, presa de la curiosidad, se concentró en escuchar. El cielo estaba despejado, como era habitual en Nápoles. Los pájaros revoloteaban sobre el pozo situado en el centro del jardín y recogían las uvas que crecían en torno a él. Los monjes recitaban ad Vesperas en la capilla y sus voces también resonaban al unísono entre las columnas. «Descartes recurre al ejemplo de la cuerda $\mathrm{ABCD}$...»-Deus in adiutorium meum intende- «si tiramos de la parte D...»-Domine ad adiuvandum me festina- «la primera parte A no se moverá de ningún modo de forma diferente...»-Gloria Patri et Filio et Spiritui Sancto- «de como ocurriría si se tira de una de las partes...»-sicut erat in principio et nunc et semper- «intermedias B o C, quedando la última D inmóvil...»-Et in secula seculorum- «Y del mismo modo, cuando siento dolor en mi pie...»-Amen-. Aunque Giambattista no acababa de comprender aquel discurso, sintió el ardor y el deseo de imitar a aquellos caballeros. Y, justo como un poderoso caballo, bien entrenado en guerra y largo tiempo después enviado al campo para que paste a voluntad, si llega a oír el sonido de la trompeta siente en sí de nuevo cómo se eleva el apetito marcial y se muestra dispuesto a ser montado por el soldado de caballería para conducirlo hacia la batalla, así él, a pesar de haberse desviado del camino recto de una temprana juventud de buena disciplina, pronto fue espoleado por su genio para recuperar el camino abandonado y emprender de nuevo su camino.

Giambattista caminó con su padre hacia la escuela de Francesco Verde (1631-1705), que enseñó derecho privado en la universidad entre 1660 y 1681, y además conoció a Felice Acquadia da Campagna (1635-1695), quien durante la conversación mencionó a Ermand Vulteius como el mejor profesor y escritor de derecho civil. Así sucedió que Giambattista se las ingenió para conseguir el libro Institutiones iuris civilis (1613) y centrar en él toda su atención. Fue mientras estudiaba Diritto Civile que Giambattista tuvo ocasión de intermediar, con la ayuda de un abogado conocido como Fabrizio Del Vecchio, a favor de su padre en una cuestión comercial en la que había sido acusado por Bartolomeo Moreschi, también librero, impresionando al tribunal hasta el punto de ser elogiado por el Consejero de la Corte, Pietro Antonio Ciavarri Eguya. ${ }^{3}$

\section{¡Menudas agallas tenía nuestro Giambattista Vico! ;El Vico de la} Reivindicación de 1729!

Las ilustres academias de enseñanza producen el más hermoso fruto para sus ciudades: por ellas los jóvenes, cuyas edades por razón de la buena sangre y la corta experiencia está henchida de confianza y nobles esperanzas, son lanzados a estudiar para alabanza y gloria. Cuando adquieren el discernimiento que les lleva a preocuparse por la utilidad llegan a ser capaces de obtener esa alabanza y gloria con honestidad por valor y mérito. ¿Pero a quién pedir ayuda?

3. F. NicOLINI, op. cit., p. 26.

Cuadernos sobre Vico 32 (2018) 
Lionardo Di Capua (1617-1695), que también vivía en la Via San Biagio dei Librai, en ocasiones se detenía ante la tienda de libros. Sucedió que, al contemplar al hombrecito tratando de leer tomos que parecían versar de graves temas, seguramente inabarcables para la edad de Giambattista, le preguntó acerca de sus intereses. El muchacho respondió amablamente a la pregunta de aquel venerable hombre y se acostumbró a sus frecuentes visitas. Juntos, el esforzado joven y el profesional, discutían muchos argumentos, y el veterano se entusiasmó ante la idea de haber encontrado un discípulo. Fue de Di Capua de quien obtuvo Giambattista una copia de los escritos de Pierre Gassendi (1592-1665). Di Capua también le consiguió a Giambattista durante una de sus visitas a la tienda una copia manuscrita del De Rerum Natura de Lucrecio a partir de la traducción de Alessandro Marchetti (1633-1714) que fue terminada en 1670. Sin embargo:

«L'intento di stampare l'opera venne ostacolato dalla politica culturale del cattolicissimo Cosimo III e dall'ingerenza dell'Inquisizione romana, tanto che Marchetti fu costretto a far circolare la traduzione solo in forma manoscritta. L'opera venne stampata solo postuma nel 1717 a Londra a cura di Paolo Rolli. Nel 1677 Marchetti ottenne l'agognata cattedra di matematica all'Università di Pisa. Dal 1691 fu membro dell'Arcadia con lo pseudonimo di Alterio Eleo». ${ }^{4}$

\section{Leer a Lucrecio fue el primer reto de Vico a la Iglesia Romana.}

A esta temprana edad, Giambattista llegó a saber de la Academia de los Investiganti, de la cual Di Capua era uno de los tres líderes más importantes. Esta academia se fundó emulando a la Academia de las Ciencias de Francia y a la Real Sociedad de Londres. Uno de los versos de Lucrecio, Vestigia lustrat, fue su lema. ${ }^{5}$ En su relación con el anciano Di Capua Giambattista llegó a hacer suyas algunas de las opiniones capuanas, como que la Iglesia Católica de Roma y el claustro de Nápoles, de hecho, habrían supuesto un pesado yugo sobre los hombros de los napolitanos y que siempre había habido un importante despertar a partir de los modos de razonamiento aristotélicos y escolásticos. Escuchando a Di Capua en sus largas conversaciones en la tienda o en el palacio de Di Capua, Giambattista, en un estado de asombro y admiración, tuvo conocimiento de la gran tradición de librepensamiento que se enseñó en Nápoles, de hombres como Bernardino Telesio, Giordano Bruno, Tommaso Campanella durante el siglo anterior, y de hombres dedi-

4. Chiara Selvaggini, Lucrezio in Italiano: Il De Rerum Natura di Alessandro Marchetti, Tesis Universidad de la Tuscia, 2008/2009.

5. Cfr. Introduction a The Autobiography of G. VICO, trad. en inglés por M.H. Fish \& T.G. Bergin, Cornell U.P., Ithaca - Londres (1944), 1975, pp. 32-33. [N.T.]. 
cados a la recuperación de la ciencia más allá de los antiguos horizontes que había fijado la tradición clerical, hombres como Galileo Galilei (1564-1652), Lord Francis Bacon de Verulam (1561-1626), Robert Boyle (1627-1691), Thomas Hobbes (15881679) y Renè Descartes (1596-1650). A pesar de que ya había oído hablar de estos filósofos, no obstante, no supo que habían sido condenados como ateos por la Iglesia de Roma hasta que se lo dijo Di Capua. En su inocente y audaz ardor juvenil no pudo hasta entonces figurarse de modo concreto el poder que la Iglesia, a través de los medios de la Inquisición, tenía en su propia tierra y época, especialmente en su ciudad. Ni siquiera se hacía idea de lo afortunado que había sido por tener la oportunidad de estar familiarizado con hombres como Lionardo Di Capua.

Resulta muy extraño pensar en todos esos autores desde una perspectiva de espacio y tiempo tan diferente a la que aquellos individuos tuvieron en relación con Giambattista. Los esquemas que trazan esta perspectiva no nos permiten realmente experimentar las mismas sensaciones que el muchacho pudo haber sentido. Todo lo que Vico escribe sobre sí mismo en su autobiografía y que podemos leer acerca de él duramente puede, ante los ojos de nuestra mente y nuestro corazón, describir la «vita» que tuvo.

Cuando Giambattista preguntó al sexagenario Di Capua cómo llegó a aprender tanto y adquirir tanto conocimiento en tantos campos de investigación y ser alguien ilustre en la ciudad, Lionardo presentó abiertamente su vida al joven. Nació y se crió en Bagnoli, un pueblecito cerca de Nápoles, una tierra que pertenecía a un Barón, a cuyos injustos deseos él, Lionardo, se opuso con duras palabras, de modo que tuvo que abandonar la tierra de su familia y establecerse en otro lugar. Di Capua había estudiado medicina, mostrando especial interés por la química, y regresó a Nápoles, donde abrió su propio estudio. Sucedió que un día en que el Virrey, habiendo perdido a un querido amigo como resultado de la incompetencia de su doctor, le pidió al Oficial Médico del Reino que revisara el acceso a la profesión médica y que estableciera un sistema idóneo en todo el reino para instruir a doctores competentes, además de formalizar las vías y medios con los que debían cumplir quienes llegasen a ser doctores. Lionardo, siendo reconocido como el más instruido en química, fue invitado a ser miembro del comité de profesionales asignados. Sin embargo, rechazó el ofrecimiento, y puso por escrito su opinión sobre el tema, censurando semejante plan por ser contrario a la práctica y la profesión médica. Recomendó que los doctores fueran libres para discutir entre ellos sus diferentes diagnósticos para elegir el que se ajustase mejor a sus propios juicios en cada caso particular. Le explicó a Giambattista que la medicina no es una ciencia exacta porque a la hora de tratar enfermedades lo que importa no es solo la pericia del doctor, sino también la posibilidad y la suerte. Entre otras historias, esta destaca especialmente porque presenta cómo los fenómenos naturales no pueden ser reducidos con tanta facilidad a fórmulas que los sinteticen. Las generalizaciones acerca de los 
fenómenos naturales no pueden ser completamente insertadas en una fórmula que los recoja. Las generalizaciones acerca de los fenómenos naturales son siempre inaceptables y uno no debe dejarse engañar por ellas. Las generalizaciones acerca de individuos son un absoluto signo de ignorancia. Vico realiza muchas generalizaciones y presuposiciones, menos en 1701, en La conspiración del Príncipe de Macchia, para el que tuvo que recorrer toda la ciudad como si fuera un reportero, y en 1716, en Vida de Antonio Caraffa, para el que tuvo que indagar en un enorme arcón de documentos cuando finalmente este llegó de Viena.

¡Ese es mi Vico!

[Traducción del inglés por Daniel Pino Sánchez]

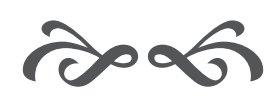

